\title{
Transition Interventions for Adolescents on Antiretroviral Therapy on Transfer From Pediatric to Adult Healthcare: A Systematic Review Protocol.
}

Olubukola Esther Jegede ( $\square$ bukolajegede.bj@gmail.com )

University of the Western Cape https://orcid.org/0000-0002-9459-0106

Brian Van Wyk

University of the Western Cape School of Public Health

\section{Protocol}

Keywords: Transition, Interventions, Pediatric, Adolescent, HIV, Antiretroviral therapy, Adherence, Retention in Care, Viral suppression

Posted Date: September 15th, 2021

DOI: https://doi.org/10.21203/rs.3.rs-876860/v1

License: (a) (i) This work is licensed under a Creative Commons Attribution 4.0 International License. Read Full License 


\section{Abstract}

Background: Adolescents living with HIV (ALHIV) experience poor health outcomes such as low retention in care, suboptimal adherence to ART, insufficient viral suppression, high morbidity and mortality. These outcomes coincide with the period during and after their transition from pediatric to adult healthcare. While some studies on transition interventions have reduced poor health outcomes among ALHIV, some have reported no significant reduction. There is a need to systematically describe, assess the quality and make recommendations regarding the effects of the transition interventions' compendium.

The proposed study aims to systematically describe interventions that guide the transition of adolescents on ART from pediatric to adult care and synthesize evidence of the effects on adherence, retention in care and viral load suppression.

Methods: A systematic review of interventions that guide the transition of adolescents on ART and report on treatment outcomes (adherence, retention in care and viral load suppression) will be conducted.

Studies will be sourced from electronic databases (PubMed, Scopus, Web of Science, Ebscohost, CINAHL, Science Direct, Google scholar and the World Health Organization's (WHO's) library database). All studies will be exported to Mendeley, and two independent reviewers will screen the titles and abstracts and the full text according to the eligibility criteria. The risk of bias will be determined using Cochrane Collaboration tool RoB 2, the Newcastle-Ottawa Scale and the Risk of Bias in Non-randomized Studies of Interventions checklists. The strength of evidence will be evaluated using the Grading of Recommendations Assessment, Development and Evaluation approach. A narrative synthesis including the description of characteristics of studies, comparison of differences and similarities between studies and description of possible trends will be carried out. If possible, homogenous studies will be subjected to a meta-analysis.

Discussion: the review findings will provide a robust knowledge base on transition interventions available to ALHIV. Assessing the strength and quality of studies will ascertain the magnitude of the effect of transition intervention outcomes and inform the development of protocols and treatment guidelines needed to achieve better health outcomes for ALHIV.

Systematic review registration: Submitted to PROSPERO on the $12^{\text {th }}$ of August 2021

\section{Background}

Poor health outcomes among adolescents living with HIV (ALHIV) are a global public health concern. Despite a global decrease of $74 \%$ in annual AIDS-related deaths among children (0-9 years) since its peak in 2003 , only a $6 \%$ decrease has been witnessed among adolescents (10-19 years) [1]. In 2019, an estimated 34,000 adolescents living with HIV died and 170,000 were newly infected [1]. Most of these deaths and new infections occurred in Sub-Saharan Africa, where the epidemic is primarily concentrated [2]. Poor outcomes such as low retention in care, low rates of viral suppression, high morbidity and 
mortality among ALHIV have been associated with suboptimal adherence to antiretroviral therapy (ART) during the adolescents' transitioning period [3-5].

Transition is defined as "the purposeful, planned movement of adolescents and young adults with chronic physical and medical conditions from child/adolescent-centered to adult-oriented healthcare systems" [6]. The transition of care happens at a critical time in adolescents' lives because adolescence is characterized by physiological and psychological developments $[7,8]$. It is widely accepted that adolescence is the period where adulthood habits are established [9]. During this time, the adolescent's HIV status are disclosed to them as they are prepared to leave the comfort of the pediatric clinic to unfamiliar territories of adult healthcare that may expose them to discrimination and stigmatization [10, 11]. Adolescents have to grapple with different truths and exposures as they are required to become increasingly self-dependent in managing their HIV condition. A structured transition is vital for continuity of care and persistent engagement in care among ALHIV.

Studies have described transition interventions from pediatric to adult healthcare in adolescents with chronic conditions such as diabetes mellitus, spina bifida, epilepsy and cystic fibrosis, but few studies described the transition to adult care for ALHIV [12-17]. The studies on structured transition interventions for ALHIV have been conducted predominantly in high-income countries [18]. While some transition interventions led to a substantial reduction in poor health outcomes among ALHIV, others have reported no significant reduction [19]. This inconsistency could result from the interventions' limited scope and generalizability [20]. Also, the effectiveness of transition interventions in adolescents living with HIV are assessed by measuring an increase in CD4 + T-lymphocyte count, viral load suppression, reduction in mortality, higher adherence to therapy and better retention in care [21,22]. Only a few studies in subSaharan Africa assessed the effectiveness of these interventions because validated tools are lacking [10]. In addition, national guidelines and clinical protocols for ALHIV are lacking in most sub-Saharan Africa countries where most ALHIVs are domiciled [23]. Yet, structured transition interventions can channel the path for ALHIV to successfully transition into adulthood [10].

Systematic reviews on impact, effectiveness and outcome evidence of transition interventions in adolescents with chronic diseases have been reported in the literature, but none of these studies focused on ALHIV during and after they transitioned to adult healthcare $[8,24,20]$. Furthermore, no systematic review has been conducted on the effects of transition interventions targeted at ALHIV. There is a need to fill this literature gap because transitioning adolescents has been associated with the worst health outcomes amongst ALHIV $[18,19]$. Systematically reviewing these interventions can provide reliable evidence from which conclusions can be drawn, and unbiased decisions can be made $[25,26]$. Also, describing and synthesizing evidence on the effects of transition interventions targeted at ALHIV will explain what works and inform reliable best practices, such as developing sound protocols that can be implemented to bring about better health outcomes for ALHIV.

The evidence synthesized from this review will provide a comprehensive knowledge-base on currently available transition interventions for ALHIV. Assessing the strength and quality of evidence will provide 
certainty in the evidence and the magnitude of effect of transition intervention outcomes for adolescents on ART. This will help inform transition policies for adolescents on ART in the right direction. This review findings can be used by policy makers and health managers to formulate protocols and treatment guidelines to improve health and treatment outcomes for ALHIV on ART. The review may also identify gaps in the literature on the evidence of effects of various transition interventions for ALHIV, and thereby inform future research agendas.

This study aims to describe interventions that guide the transition of adolescents on ART from pediatric to adult care with a view to synthesizing evidence of the effects of the interventions on adherence, retention in care and viral load suppression.

The specific objectives of the study are: (1) to describe transition interventions designed to improve treatment outcomes of adolescents living with HIV on transfer from pediatric to adult healthcare; (2) to appraise the quality of studies on transition interventions on adolescents living with HIV; and (3) to make recommendations for transition interventions by assessing the strength of evidence.

\section{Methods}

This systematic review protocol follows the preferred reporting items for systematic reviews and metaanalysis protocols (PRISMA-P 2015) guidelines (see Additional file 1: PRISMA-P 2015 Checklist).

\section{Eligibility Criteria}

The PICOT mnemonic standing for population, intervention, comparative, outcomes and time will guide the definition of inclusion and exclusion criteria.

\section{Study design}

Randomized control trials, non-randomized control trials as well as observational studies will be sourced for in this review.

\section{Participants}

We will include studies whose Population of interest include adolescents aged 10-19 years, living with HIV and on ART.

\section{Interventions and comparators}

The Intervention of interest will be transition interventions for adolescents on HIV treatment (ART). There will be no Comparison as observational studies will be included in this review.

Transition interventions are structured and designed to improve the health outcomes of adolescents and young adults with chronic health challenges pre-, during and post-transfer from child/adolescent healthcare facilities to adult healthcare facilities. Pre-transition intervention happens within the 
child/adolescent facilities and is run by multidisciplinary healthcare professionals. It starts with disclosure of status and involves transition preparedness, such as addressing what transition is and its barriers, for example, anxiety and fear of transition, which can be measured by using transition readiness scale $[22,27]$. This intervention could also focus on providing skills and discussions on adherence to ART, sexually-oriented relationships and coping tactics [8]. These interventions flow into the during transition interventions. The transition interventions targeted at when the transition will occur could include communication between pediatric/adolescent and adult providers. This communication includes adolescents being introduced to and having a full consultation with adult healthcare personnel within the pediatric/adolescent clinic and assistance provided for adolescents to overcome system-level barriers such as insurance changes or social services [27, 28]. These interventions also involve ALHIV being taught self-efficacy and psychosocial skills in peer/family/community support groups as they get transferred to adult facility at this stage.

Post-transition interventions occur in the adult clinics and are more focused on assessing transition outcomes. Transition experiences of ALHIV are gauged. Also, post-transition immunological and virological measurements are taken. To inform better strategies to transition ALHIV and examine if the transition was successful or not [29].

\section{Outcomes}

The primary Outcomes of interest will be adherence to ART, retention in care, or viral load suppression. All secondary outcomes such as psychosocial well-being and self-efficacy will also be considered. To ascertain the success of the transition interventions, the study will consider transition outcomes such as percentage/rate of ALHIV recorded as a loss to follow up or retained in care over a period and mortality rate post-transition. It will also consider immunological and virological outcomes such as CD4 count and viral load measurement post-transition as reported in the studies.

\section{Timeframe}

The Timeframe for the inclusion of published articles will be 2000 to 2021 .

\section{Setting}

There will be no restriction on settings or geographical location.

\section{Further inclusion criteria}

Grey literature and quantitative studies published in peer-reviewed journals in any language will be included. All languages will be considered to avoid publication bias. The studies should report quantitative results on ART adherence measurement, retention in care, viral suppression, psychosocial well-being or self-efficacy.

\section{Exclusion criteria}


Literature reviews, protocols, systematic reviews and meta-analyses will be excluded. Qualitative studies will also be excluded because they do not report quantitative outcomes of interest. Studies that do not focus primarily on transition intervention but just mentioned transition in passing will be excluded.

\section{Information sources and search strategy}

We will conduct a comprehensive systematic search using predetermined search strings on electronic databases such as PubMed, Scopus, Web of Science, Ebscohost, CINAHL, Science Direct, Google scholar and the World Health Organization's (WHO's) library database. (See Additional file 2: Draft for the database search strategies). Likewise, the reference list of included studies will be hand-searched to identify additional eligible studies.

\section{Study selection}

Two independent reviewers will screen the title and abstracts of the studies identified according to the PICOT criteria: (1) the study population include adolescents (10-19 years) living with HIV; (2) involves a transition intervention (transfer from pediatric to adult HIV care program); (3) reports quantitative (outcome) results of the intervention; and (4) the timeframe for the inclusion of published articles will be 2000 to 2021. Full texts of the eligible studies will be retrieved and reviewed by the two reviewers. A third reviewer will resolve a conflict of judgment between the two reviewers when this is not resolved through discussion. The Preferred Reporting Items stipulate for Systematic Reviews and Meta-Analyses protocols (PRISMA-P) statement [30] will inform the writing of the protocol and the study results will be reported according to the Preferred Reporting Items stipulate for Systematic Reviews and Meta-Analyses (PRISMA) 2020 statement [31]. The PRISMA 2020 statement consists of 27-item checklists and a four-phase flow diagram, which will be adhered to for reporting and the four-phase flow diagram for screening studies.

\section{Data extraction}

A data extraction form will be designed to gather information on the author and year of publication, study population, intervention description, study design/outcome, outcomes' measurement approach, result, level of evidence and intervention effect. This helps to synthesize relevant information from the individual studies in a standard and transparent way. The information extracted is presented in a table clearly and concisely. The data extraction form will be piloted in at least five transition intervention studies on ALHIV before finalization. To organize the data extraction form, Microsoft Excel software applications will be used.

\section{Outcomes of interest for adolescents on antiretroviral therapy}

Extant literatures [32-36] have identified the three primary outcomes of interest among ALHIV to be adherence to ART, retention in care and viral load suppression.

Adherence 
Adherence can be defined as the process by which HIV patients take their medication (antiretroviral drugs) as prescribed [37]. Adherence to ART of at least 95\% (for a daily single-pill regimen - 14 nonsequential missed doses over a year) is recommended as optimal adherence. At the same time, anything below is considered a suboptimal/poor adherence to ART [38].

\section{Method of adherence measurement}

Varied adherence to ART measurements exists in the literature, and these include viral load, self-report, pill count, home record, pharmacy refill, healthcare provider assessment, or electronic monitoring [39]. Data on the listed method of adherence measurement will be considered in this review.

\section{Viral load suppression}

According to [40], viral load suppression is defined as "suppressing or reducing the function and replication of a virus." Hence, viral load measurement is used to monitor treatment effectiveness in HIV patients on ART [36]. According to the Abbott platform, an undetectable viral load occurs when viral load reads $<40$ copies $/ \mathrm{ml}$ and a detectable viral load at $\geq 40 \mathrm{copies} / \mathrm{ml}$ [41]. Virological failure occurs when viral load is $>1000$ copies $/ \mathrm{ml}$ after two consecutive viral load tests in 3 months, which involves having adherence support and being on ART for at least 6 months [42].

\section{Retention in care}

The World Health Organization defines retention in care as "the continuous engagement from diagnosis in a package of prevention, treatment, support and care services [36]." Retention in care can be measured by considering missed visits, appointment adherence, visit constancy and gaps in care, as indicators [43]. Low retention in care has been associated with non-viral suppression and a high mortality rate [44-46].

\section{Risk of bias assessment}

Two researchers will screen and evaluate the quality of each selected research independently to ensure accuracy and generalizability [47]. Checklists such as the Cochrane Collaboration tool RoB 2 will be used to assess the risk of bias in included randomized control trial studies [48]. The Newcastle-Ottawa Scale checklist will be used to assess the risk of bias in included prospective observational studies [49]. The Risk of Bias in Non-randomized Studies - of Interventions (ROBINS-I) will be used to assess the risk of bias in included non-randomized intervention studies [50].

\section{Effect measures}

We will consider effect size measurements such as the relative risk, risk difference and confidence intervals $(\mathrm{Cl})$ from the individual included studies and for each of the outcome of interest. This will inform the overall magnitude of effect and evidence confidence.

\section{Data Analysis}


The data extracted in step 5 will be imported to SPSS statistical package for analysis. If there is high heterogeneity in the included studies such that a meta-analysis is impossible, the present study will adopt a narrative synthesis. The narrative synthesis will structurally summarize and describe the characteristics of the studies: study population, intervention description and study design/outcome, their findings and quality [47]. The included studies' findings will highlight their direction and effect size, effect consistency and the strength of evidence for the effect [47]. While for the quality, the study will only include studies with low or moderate risk of bias to improve the trustworthiness of the synthesis [51]. Studies included will be compared for similarities and differences, while possible trends will also be described.

If meta-analysis is possible, it will be done using Stata statistical package and will be focused on randomized control trials. The study will use a random-effects meta-analysis because, according to [47], "it allows the true effects underlying the studies to differ and thus accounts for unexplained heterogeneity between studies." The study will pool estimates to obtain a summary estimate, with $95 \% \mathrm{Cl}$. The $\chi 2$ test will be performed to test for heterogeneity, and $\mathrm{I}^{2}$ statistic to assess the degree of homogeneity [52]. If the study finds more than ten studies included, we will use a funnel plot and an Egger test to assess publication bias [53]. If there are sufficient data, we will consider doing a subgroup analysis on age intervals, sex, study designs and model of transition interventions.

\section{Certainty assessment}

Two reviewers will individually ascertain the overall quality of evidence for the primary outcomes of interest. The strength and certainty of evidence from each study included will be evaluated according to the GRADE system [54]. The GRADE system provides the platform to assess the quality of evidence for each outcome on five different domains which are: risk of bias, consistency; directness; precision; and publication bias. The presence of risk of bias (study limitations), inconsistency, indirectness, imprecision or publication bias leads to lowering the quality of evidence while large magnitude of effect, opposing plausible confounders and dose-response gradient can increase the quality of evidence [54]. Confidence in the effect estimate for each outcome will be ascertained independently by the two reviewers as high, moderate, low or very low. High denotes sound confidence in the true effect lying close to that of the estimate of the effect. Moderate denotes moderate confidence in the true effect lying close to that of the estimate of the effect i.e., there is a possibility for the true effect to be substantially different from the estimate effect. Low denotes limited confidence in the true effect lying close to that of the estimate of the effect i.e., true effect may be substantially different from estimate effect. Very Low denotes very little confidence in the true effect lying close to that of the estimate of the effect as the true effect is very likely to be different from the estimate effect [55]. A third reviewer will resolve a conflict of judgment between the two reviewers when this is not resolved through discussion. A detailed summary of findings table will be included in the actual review paper.

\section{Discussion}


Adolescents living with HIV represent the group with HIV with the worst health outcomes (low retention in care, suboptimal adherence to ART and insufficient viral suppression). These worst health outcomes are exacerbated during and after they transition from pediatric to adult healthcare. A structural transition intervention, when implemented, has been reported to facilitate better health outcomes among ALHIV. This systematic review aims to describe interventions that guide the transition of adolescents on ART from pediatric to adult care and synthesize evidence of the effects of the interventions on adherence to ART, retention in care and viral load suppression.

The strength of this systematic review protocol lies in its intention to appraise the quality of the studies, assess the strengths of evidence and make recommendations that can lead to improvements in health and treatment outcomes for ALHIV on ART. This is further strengthened by searching eight databases through the formulation of search strategy based on keywords such as "transition", "intervention", "adolescent", "HIV", "antiretroviral therapy", "retention", "viral suppression", "pediatric", "adult" and "healthcare". The study will also adhere to the preferred reporting items for systematic reviews and metaanalysis protocols (PRISMA-P 2015) guidelines. Focusing on studies with low or moderate risk of bias helps strengthen and improve the trustworthiness of the synthesis [51].

The results from this study will provide the knowledge base for the compendium of transition interventions that exist for ALHIV, which will help inform policies that affect them in the right direction. The results also have the potential to guide policymakers and health managers in developing sound and generalizable structural transition protocols for ALHIV.

\section{Abbreviations}

ART Antiretroviral Therapy

HIV Human Immunodeficiency Virus

ALHIV Adolescents Living with Human Immunodeficiency Virus

UNICEF United Nations International Children's Emergency Fund

UNAIDS United Nations Programme on HIV/AIDS

CDC Centers for Diseases Control and Prevention

PRISMA Preferred Reporting Items for Systematic Reviews and Meta-Analyses

PRISMA-P Preferred Reporting Items for Systematic Reviews and Meta-Analyses Protocol

CD4+ Cluster of Differentiation 4

ROBINS-I Risk of Bias in Non-randomized Studies - of Interventions 
RoB 2 Risk of Bias 2

\section{Declarations}

\section{Acknowledgments}

Ms. Karen Cook, Senior Librarian: Community and Health Sciences Branch Library, at the University of the Western Cape, for her guide in developing the search strategy as well as the databases.

\section{Authors' Contributions}

BvW presented the idea to OEJ. OEJ developed the protocol research questions and study methods and contributed meaningfully to the drafting and editing. She also approved the final manuscript. BvW aided in developing the research questions and study methods, and contributed meaningfully to the drafting and editing and approved the final manuscript.

\section{Funding}

self-funded

\section{Availability of data and materials}

Not applicable.

\section{Ethics approval and consent to participate}

Not applicable.

\section{Consent for publication}

Not applicable.

\section{Competing interests}

The authors declare that they have no competing interests.

\section{References}

1. United Nations Children's Fund. HIV statistics - global and regional trends - UNICEF data. UNICEF. 2020. https://data.unicef.org/topic/hivaids/global-regional-trends/. Accessed 3 August 2021.

2. United Nations Programme on HIV/AIDS. Data 2020. Programme on HIV/AIDS, 1-248. UNAIDS; 2020. https://www.unaids.org/en/resources/documents/2020/unaidsdata\%0Ahttp://www.unaids.org/sites/default/files/media_asset/20170720_Data_book_2017_en.pdf. Accessed 17 July 2021. 
3. Badejo OA, Menson WN, Sam-Agudu NA, Pharr J, Erekaha S, Bruno T, Nwanne G, Ogunsola O, llozumba J, Busari O, Ezeanolue EE. Pediatric to adult healthcare transitioning for adolescents living with HIV in Nigeria: A national survey. PLoS One. 2018 Jun 12;13(6):e0198802.

4. Jerene D, Abebe W, Taye K, Ruff A, Hallstrom I. Adolescents living with HIV are at higher risk of death and loss to follow up from care: Analysis of cohort data from eight health facilities in Ethiopia. PloS one. 2019 Oct 17;14(10):e0223655.

5. Pinzón-Iregui MC, Ibanez G, Beck-Sagué C, Halpern M, Mendoza RM. “... like becauseyou are a grownup, you do not need help": Experiences of transition frompediatric to adult care among youth with perinatal HIV infection, their caregivers, and health care providers in the Dominican Republic. Journal of the InternationalAssociation of Providers of AIDS Care (JIAPAC). 2017 Nov; 16(6):579-87.

6. Blum RW, Garell D, Hodgman CH, Jorissen TW, Okinow NA, Orr DP, Slap GB.Transition from childcentered to adult health-care systems for adolescents with chronic conditions: a position paper of the Society for Adolescent Medicine.Journal of Adolescent Health. 1993 Nov 1;14(7):570-6.

7. Adejumo OA, Malee KM, Ryscavage P, Hunter SJ, Taiwo BO. Contemporary issues onthe epidemiology and antiretroviral adherence of HIV-infected adolescents in sub-Saharan Africa: a narrative review. Journal of the International AIDSSociety. 2015 Jan;18(1):20049.

8. Campbell F, Biggs K, Aldiss SK, O'Neill PM, Clowes M, McDonagh J, While A, GibsonF. Transition of care for adolescents from paediatric services to adult healthservices. Cochrane Database of Systematic Reviews. 2016(4).

9. Sawyer SM, Azzopardi PS, Wickremarathne D, Patton GC. The age of adolescence. The Lancet Child \& Adolescent Health. 2018 Mar 1;2(3):223-8.

10. Njuguna I, Beima-Sofie K, Mburu C, Mugo C, Black DA, Neary J, Itindi J, Onyango A,Slyker J, Oyiengo $\mathrm{L}$, John-Stewart G. Managing the transition from paediatric toadult care for HIV, Kenya. Bulletin of the World Health Organization. 2019 Dec1;97(12):837.

11. Tanner AE, Philbin MM, DuVal A, Ellen J, Kapogiannis B, Fortenberry JD, AdolescentTrials Network for HIV/AIDS Interventions. Transitioning HIV-positive adolescents to adult care: lessons learned from twelve adolescent medicine clinics. Journal of pediatric nursing. 2016 Sep 1;31(5):537-43.

12. Betz CL. Transition of adolescents with special health care needs: review and analysis of the literature. Issues in comprehensive pediatric nursing. 2004 Jan 1;27(3):179- 241.

13. Brumfield K, Lansbury G. Experiences of adolescents with cystic fibrosis during their transition from paediatric to adult health care: a qualitative study of youngAustralian adults. Disability and rehabilitation. $2004 \mathrm{Feb}$ 18;26(4):223-34.

14. Freed GL, Hudson EJ. Transitioning children with chronic diseases to adult care: current knowledge, practices, and directions. The Journal of pediatrics. 2006 Jun1;148(6):824-7.

15. Lewis-Gary MD. Transitioning to adult health care facilities for young adults with achronic condition. Pediatric nursing. 2001 Sep 1;27(5):521.

16. Maturo D, Powell A, Major-Wilson H, Sanchez K, De Santis JP, Friedman LB. Development of a protocol for transitioning adolescents with HIV infection toadult care. Journal of Pediatric Health 
Care. 2011 Jan 1;25(1):16-23.

17. Por J, Golberg B, Lennox V, Burr P, Barrow J, Dennard L. Transition of care: health careprofessionals' view. Journal of nursing management. 2004 Sep;12(5):354-61.

18. Njuguna IN, Beima-Sofie K, Mburu CW, Mugo C, Neary J, Itindi J, Onyango A, Richardson BA, Means AR, Sharma M, Weiner BJ. Adolescent transition to adultcare for HIV-infected adolescents in Kenya (ATTACH): study protocol for a hybrid effectiveness-implementation cluster randomised trial. BMJ open. 2020 Dec 1;10(12):e039972.

19. Judd, A., Sohn, A.H. and Collins, I.J., 2016. Interventions to improve treatment, retention and survival outcomes for adolescents with perinatal HIV-1 transitioning to adultcare: moving on up. Current opinion in HIV and AIDS, 11(5), pp.477-486.

20. Gabriel P, McManus M, Rogers K, White P. Outcome evidence for structured pediatric to adult health care transition interventions: a systematic review. The Journal of pediatrics. 2017 Sep 1;188:263-9.

21. Lolekha R, Boon-yasidhi V, Na-Nakorn Y, Manaboriboon B, Vandepitte WP, Martin M, Tarugsa J, Nuchanard W, Leowsrisook P, Lapphra K, Suntarattiwong P. TheHappy Teen programme: a holistic outpatient clinic-based approach to prepareHIV-infected youth for the transition from paediatric to adult medical careservices in Thailand. Journal of the International AIDS Society. 2017 May;20:21500.

22. Wiener L, Battles H, Ryder C, Zobel M. Transition from a pediatric HIV intramural clinical research program to adolescent and adult community-based care services: assessing transition readiness. Social work in health care. 2007 Sep 27;46(1):1-9.

23. Dahourou DL, Gautier-Lafaye C, Teasdale CA, Renner L, Yotebieng M, Desmonde S,Ayaya S, Davies MA, Leroy V. Transition from paediatric to adult care ofadolescents living with HIV in sub-Saharan Africa: challenges, youth-friendly models, and outcomes. Journal of the International AIDS Society. 2017May;20:21528.

24. Chu PY, Maslow GR, von Isenburg M, Chung RJ. Systematic review of the impact of transition interventions for adolescents with chronic illness on transfer from pediatric to adult healthcare. Journal of pediatric nursing. 2015 Sep 1;30(5):e19-27.

25. Antman EM, Lau J, Kupelnick B, Mosteller F, Chalmers TC. A comparison of results of meta- analyses of randomized control trials and recommendations of clinical experts: treatments for myocardial infarction. Jama. 1992 Jul 8;268(2):240-8.

26. Oxman AD, Guyatt GH. The science of reviewing research a. Annals of the New YorkAcademy of Sciences. 1993 Dec;703(1):125-34.

27. Fair CD, Sullivan K, Gatto A. Best practices in transitioning youth with HIV: perspectives of pediatric and adult infectious disease care providers. Psychology, Health \& Medicine. 2010 Oct 1;15(5):515-27.

28. Gilliam PP, Ellen JM, Leonard L, Kinsman S, Jevitt CM, Straub DM. Transition ofadolescents with HIV to adult care: characteristics and current practices of theadolescent trials network for HIV/AIDS interventions. Journal of the Association of Nurses in AIDS Care. 2011 Jul 1;22(4):283-94. 
29. Hussen SA, Chahroudi A, Boylan A, Camacho-Gonzalez AF, Hackett S, Chakraborty R. Transition of youth living with HIV from pediatric to adult-oriented healthcare: a review of the literature. Future virology. 2014 Oct;9(10):921-9.

30. Moher D, Shamseer L, Clarke M, Ghersi D, Liberati A, Petticrew M, Shekelle P, Stewart LA. Preferred reporting items for systematic review and meta-analysis protocols (PRISMA-P) 2015 statement. Systematic reviews. 2015 Dec;4(1):1-9.

31. Page MJ, McKenzie JE, Bossuyt PM, Boutron I, Hoffmann TC, Mulrow CD, Shamseer L, Tetzlaff JM, AkI EA, Brennan SE, Chou R. The PRISMA 2020 statement: an updated guideline for reporting systematic reviews. Bmj. 2021 Mar 29;372.

32. lacob SA, lacob DG, Jugulete G. Improving the adherence to antiretroviral therapy, adifficult but essential task for a successful hiv treatment-clinical points of viewand practical considerations. Frontiers in pharmacology. 2017 Nov 23;8:831.

33. Mukumbang FC, Van Belle S, Marchal B, Van Wyk B. 'At this [adherence] club, we are a family now': A realist theory-testing case study of the antiretroviral treatmentadherence club, South Africa. Southern African journal of HIV medicine. 2019 Jan 1;20(1):1-4.

34. Nachega JB, Parienti JJ, Uthman OA, Gross R, Dowdy DW, Sax PE, Gallant JE,Mugavero MJ, Mills EJ, Giordano TP. Lower pill burden and once-daily antiretroviral treatment regimens for HIV infection: a meta-analysis of randomized controlled trials. Clinical infectious diseases. 2014 May 1;58(9):1297307.

35. Phelps BR, Hathcock SJ, Werdenberg J, Schutze GE. Experiencing antiretroviral adherence: Helping healthcare staff better understand adherence to paediatricantiretrovirals. Journal of the International AIDS Society. 2010 Dec;13(1):1-4.

36. World Health Organization. Retention in HIV programmes: defining the challenges andidentifying solutions: meeting report, 13-15 September 2011.

37. Vrijens B, De Geest S, Hughes DA, Przemyslaw K, Demonceau J, Ruppar T, Dobbels F, Fargher E, Morrison V, Lewek P, Matyjaszczyk M. A new taxonomy fordescribing and defining adherence to medications. British journal of clinical pharmacology. 2012 May;73(5):691-705.

38. Neupane S, Dhungana GP, Ghimire HC. Adherence to antiretroviral treatment and associated factors among people living with HIV and AIDS in CHITWAN, Nepal. BMC public health. 2019 Dec;19(1):1-9.

39. Hudelson C, Cluver L. Factors associated with adherence to antiretroviral therapy amongadolescents living with HIV/AIDS in low-and middle-income countries: a systematic review. AIDS care. $2015 \mathrm{Jul}$ 3;27(7):805-16.

40. Boskey, E. (2020). What viral suppression has to do with HIV therapy. https://www.verywellhealth.com/viral-suppression-3132658. Accessed 19 July 2021.

41. Bermudez LG, Ssewamala FM, Neilands TB, Lu L, Jennings L, Nakigozi G, Mellins CA, McKay M, Mukasa M. Does economic strengthening improve viral suppressionamongadolescents living with HIV? Results from a cluster randomized trial inUganda. AIDS and Behavior. 2018 Nov;22(11):376372. 
42. World Health Organization. 2010 progress report. World Health Organization; 2010. www.who.int/hiv Accessed 3 August 2021.

43. Mugavero MJ, Davila JA, Nevin CR, Giordano TP. From access to engagement:measuring retention in outpatient HIV clinical care. AIDS patient care and STDs. 2010 Oct 1;24(10):607-13.

44. Giordano TP, Gifford AL, White Jr AC, Almazor ME, Rabeneck L, Hartman C, BackusLI, Mole LA, Morgan RO. Retention in care: a challenge to survival with HIV infection. Clinical infectious diseases. 2007 Jun 1;44(11):1493-9.

45. Mugavero MJ, Lin HY, Willig JH, Westfall AO, Ulett KB, Routman JS, Abroms S, Raper JL, Saag MS, Allison JJ. Missed visits and mortality among patients establishing initial outpatient HIV treatment. Clinical Infectious Diseases. 2009Jan 15;48(2):248-56.

46. Mugavero MJ, Westfall AO, Cole SR, Geng EH, Crane HM, Kitahata MM, MathewsWC, Napravnik S, Eron JJ, Moore RD, Keruly JC. Beyond core indicators of retention in HIV care: missed clinic visits are independently associated with all- cause mortality. Clinical Infectious Diseases. $2014 \mathrm{Nov}$ 15;59(10):1471-9.

47. Muka T, Glisic M, Milic J, Verhoog S, Bohlius J, Bramer W, Chowdhury R, Franco OH.A 24-step guide on how to design, conduct, and successfully publish a systematic review and meta-analysis in medical research. European journal of epidemiology.2020 Jan;35(1):49-60.

48. Higgins JP, Sterne JA, Savovic J, Page MJ, Hróbjartsson A, Boutron I, Reeves B, Eldridge S. A revised tool for assessing risk of bias in randomized trials. Cochrane database of systematic reviews. 2016;10(Suppl 1):29-31.

49. Wells GA, Shea B, O'Connell D, Peterson J, Welch V, Losos M, Tugwell P. The Newcastle-Ottawa Scale (NOS) for assessing the quality of nonrandomized studies in meta-analyses.

50. Sterne JA, Hernán MA, Reeves BC, Savović J, Berkman ND, Viswanathan M, Henry D, Altman DG, Ansari MT, Boutron I, Carpenter JR. ROBINS-I: a tool for assessing risk of bias in non-randomised studies of interventions. bmj. 2016 Oct 12;355.

51. Popay J, Roberts H, Sowden A, Petticrew M, Arai L, Rodgers M, Britten N, Roen K, Duffy S. Guidance on the conduct of narrative synthesis in systematic reviews. A product from the ESRC methods programme Version. 2006 Apr 1;1:b92.

52. Roomaney RA, van Wyk B, Turawa EB, Pillay-van Wyk V. Prevalence of multimorbidity in South Africa: a systematic review protocol. BMJ open. 2020Dec 1;10(12):e042889.

53. Egger M, Smith GD, Schneider M, Minder C. Bias in meta-analysis detected by a simple,graphical test. Bmj. 1997 Sep 13;315(7109):629-34.

54. Guyatt GH, Oxman AD, Schünemann HJ, Tugwell P, Knottnerus A. GRADE guidelines:a new series of articles in the Journal of Clinical Epidemiology. Journal of clinical epidemiology. $2011 \mathrm{Apr}$ 1;64(4):380-2.

55. Balshem H, Helfand M, Schünemann HJ, Oxman AD, Kunz R, Brozek J, Vist GE, Falck-Ytter $Y$, Meerpohl J, Norris S, Guyatt GH. GRADE guidelines: 3. Rating the quality of evidence. Journal of clinical epidemiology. 2011 Apr 1;64(4):401-6.

Page 14/15 


\section{Supplementary Files}

This is a list of supplementary files associated with this preprint. Click to download.

- AdditionalFile1and2.docx 